\title{
CONDITIONAL EXPECTATIONS RELATIVE TO A PRODUCT STATE AND THE CORRESPONDING STANDARD POTENTIALS
}

\author{
HUZIHIRO ARAKI
}

\begin{abstract}
For a lattice system with a finite number of Fermions and spins on each lattice point, conditional expectations relative to an even product state (such as Fermion Fock vacuum) are introduced and the corresponding standard potential for any given dynamics, or more generally for any given time derivative (at time 0 ) of strictly local operators, is defined, with the case of the tracial state previously treated as a special case. The standard potentials of a given time derivative relative to different product states are necessarily different but they are shown to give the same set of equilibrium states, where one can compare states satisfying the variational principle (for translation invariant states) or the local thermodynamical stability or the Gibbs condition, all in terms of the standard potential relative to different even product states.
\end{abstract}

\section{IntRoduction}

Conditional expectations from a $C^{*}$-algebra to its subalgebras relative to the unique tracial state have been recently used as a basic tool for the formulation of equilibrium statistical mechanics of Fermion lattice systems and for the equivalence proof of the KMS condition and the variational principle for translation invariant states [2]. The main role of the conditional expectations there, apart from their use as an effective tool of various proofs, is the unique association of the standard potential (characterized by vanishing of appropriate conditional expectations) for a given dynamics.

In the present work, we generalize the definitions and results in [2] by introducing $\omega$-conditional expectations relative to an even product state $\omega$. A mathematical difference from the slice map treated in mathematical literatures is the mutual noncommutativity of factor subalgebras, relative to which the state $\omega$ has the product property. The algebra under consideration is somewhat generalized from the one in [2] to a graded $C^{*}$ algebra with a graded commutation relations, which may include simultaneously both Fermion creation and annihilation operators and spin operators at each lattice point, as long as the local algebra at each lattice point is a full matrix algebra (i.e. a finite dimensional factor), excluding a possibility for Boson creation and annihilation operators.

All results in [2] as well as those in [3] hold also for $\omega$-standard potentials relative to a product state $\omega$. For different choices of $\omega$ and a fixed dynamics, they provide examples of equivalent potentials. Each of characterizations of equilibrium states in terms of the $\omega$-standard potential, such as the variational principle, the Gibbs condition and the LTS condition, gives the same set of equilibrium state for any different choice of the product state $\omega$ (Theorems 5.1 and 7.2). 
As an immediate consequence, mutual equivalence of the KMS condition, the dKMS condition, the Gibbs condition, the LTS condition, and the variational principle (the last one only for translation invariant states), which is derived in [2] and [3] (Theorems A, B, 7.5, 7.6, and Proposition 12.1 of [2] and Theorems 1, 2, 3 and Corollary 4 of $[3])$ for $(\tau-)$ standard potentials under (minimal) assumptions on dynamics, holds also for the general $\omega$-standard potentials.

The paper is organized as follows. The graded algebra and its graded commutation relations along with results on commutants (Theorem 2.4 and 2.5) and intersections (Theorem 2.2) are described and proved in Section 2. The $\omega$-conditional expectations relative to a product state $\omega$ along with their basic properties (Theorems 3.1 and 3.2) are given in Section 3. The $\omega$-standard potentials for a given dynamics are introduced in Section 4 with a use of the $\omega$-conditional expectations. The Gibbs and LTS conditions are described in terms of the $\omega$-standard potentials in Section 5. Translation invariance is introduced in Section 6 and the variational principle is discussed in Section 7. All results and their proofs in [2] and [3] can be carried over to the present generalized situation (Theorems 4.1, 4.2, 5.1, 6.1, $6.2,6.3$, and 7.1). Comparison of the $\omega$-standard potentials for different choices of $\omega$ (the tracial state and the vacuum state of a Fermion lattice system) are made for one-body and two-body potentials in Section 8. The $\omega$-conditional expectations for a non-even product state is discussed in Section 9. A necessary and sufficient condition for a subset $I$ of the lattice is given for the existence of the $\omega$-conditional expectation onto the subalgebra for the subset $I$ in the case of non-even $\omega$ (Theorem $9.1)$.

\section{Algebra}

We consider a $C^{*}$-algebra $\mathcal{A}$ equipped with the following structure, modeled after Fermion and spin lattice systems.

(a) Local structure.

For each point $i$ of a lattice $L=\mathbb{Z}^{\nu}$, there corresponds a subalgebra $\mathcal{A}_{i}$ of $\mathcal{A}$, which is isomorphic to a full matrix algebra of $d \times d$ matrices, $d$ independent of $i$ (independence needed for lattice translation automorphisms).

For each subset $I$ of $L, \mathcal{A}(I)$ denotes the $C^{*}$-subalgebra of $\mathcal{A}$ generated by $\mathcal{A}_{i}, i \in I . \mathcal{A}(L)$ is assumed to be $\mathcal{A}$.

In most part of this work except Sections 2 and 9, we assume the existence of a representation of the group $L$ by automorphisms $\tau_{k}$ of $\mathcal{A}, k \in L$, such that $\tau_{k}\left(\mathcal{A}_{i}\right)=\mathcal{A}_{i+k}$. Then

$$
\tau_{k}(\mathcal{A}(I))=\mathcal{A}(I+k), \quad I+k=\{i+k ; i \in I\} .
$$

(b) Graded structure.

There exists an involutive $C^{*}$-automorphism $\Theta$ of $\mathcal{A}$ such that

$$
\begin{gathered}
\Theta(\mathcal{A}(I))=\mathcal{A}(I), \\
\Theta \tau_{k}=\tau_{k} \Theta, \quad(k \in L) .
\end{gathered}
$$

Then any $A \in \mathcal{A}$ splits uniquely as a sum of even and odd elements $A_{+}$and $A_{-}$:

$$
\begin{gathered}
A=A_{+}+A_{-}, \\
A_{ \pm}=(1 / 2)(A \pm \Theta(A)), \quad \Theta\left(A_{ \pm}\right)= \pm A_{ \pm} .
\end{gathered}
$$


Accordingly, $\mathcal{A}$ and the subalgebras $\mathcal{A}(I)$ split as a sum of even and odd parts which have a trivial (i.e. zero) intersection:

$$
\begin{gathered}
\mathcal{A}=\mathcal{A}_{+}+\mathcal{A}_{-}, \quad \mathcal{A}_{ \pm}=\{A \in \mathcal{A} ; \Theta(A)= \pm A\}, \\
\mathcal{A}(I)=\mathcal{A}(I)_{+}+\mathcal{A}(I)_{-}, \quad \mathcal{A}(I)_{ \pm}=\mathcal{A}(I) \cap \mathcal{A}_{ \pm} .
\end{gathered}
$$

The following graded commutation relations hold: if $I \cap J=\emptyset, A_{\sigma} \in \mathcal{A}(I)_{\sigma}$, and $B_{\sigma^{\prime}} \in \mathcal{A}(J)_{\sigma^{\prime}}\left(\sigma, \sigma^{\prime}= \pm\right)$, then

$$
\begin{gathered}
A_{\sigma} B_{\sigma^{\prime}}=\epsilon\left(\sigma, \sigma^{\prime}\right) B_{\sigma^{\prime}} A_{\sigma}, \\
\epsilon\left(\sigma, \sigma^{\prime}\right)= \begin{cases}-1, & \text { if } \sigma=\sigma^{\prime}=-, \\
+1, & \text { otherwise. }\end{cases}
\end{gathered}
$$

Namely, odd elements of disjoint regions anticommute, while other pairs of even and odd elements commute. The graded commutation relations hold for any pair of disjoint I and $\mathrm{J}$ if they hold for a pair of disjoint one-point sets because $\mathcal{A}(I)$ is generated by $\mathcal{A}_{i}, i \in I$.

We use the notation $I \subset \subset L$ to mean that $I$ is a finite subset of $L$. Then $|I|$ denotes the number of points in $I$, sometimes called the volume of $I$. We denote

$$
\mathcal{A}_{0}=\cup_{I \subset \subset L} \mathcal{A}(I) .
$$

It is a dense $*$-subalgebra of $\mathcal{A}$.

Lemma 2.1. For $I \subset \subset L, \mathcal{A}(I)$ is isomorphic to a full matrix algebra of $d^{|I|} \times d^{|I|}$ matrices. For an infinite subset I of $L, \mathcal{A}(I)$ is a UHF algebra of type $d^{\infty}$. In paticular, $\mathcal{A}(I)$ is simple for all I. As a special case, $\mathcal{A}$ is simple.

Proof. First we prove the first assertion inductively for increasing $|I|$. For this, it is enough to consider disjoint finite subsets $I$ and $J$ of $L$ and to prove that $\mathcal{A}(I \cup J)$ satisfies the first assertion if $\mathcal{A}(I)$ and $\mathcal{A}(J)$ do.

Since any $*$-automorphism of a type I factor is inner, there exists a unitary $u \in \mathcal{A}(I)$ satisfying $\operatorname{Ad} u=\Theta$ on $\mathcal{A}(I)$. By adjusting a constant multiple of modulus 1 , we may assume $u^{2}=1$ due to $\Theta^{2}=i d$. (Then $\pm u$ are the only selfadjoint unitaries in $\mathcal{A}(I)$ which implement $\Theta$ on $\mathcal{A}(I)$.) By $\Theta(u)=u^{3}=u$, we have $u \in \mathcal{A}(I)_{+}$and hence $u \in \mathcal{A}(J)^{\prime}$.

Consider the mapping

$$
\pi: \quad A \in \mathcal{A}(J) \longrightarrow \pi(A)=A_{+}+u A_{-} .
$$

It is readily seen that $\pi$ is a unital $*$-homomorphism. Since a full matrix algebra is simple, $\pi$ is an isomorphism. Furthermore, $\pi(\mathcal{A}(J)) \in \mathcal{A}(I)^{\prime}$ due to $A d u=\Theta$ on $\mathcal{A}(I)$. Therefore the $C^{*}$-subalgebra of $\mathcal{A}$ generated by $\mathcal{A}(I)$ and $\pi(\mathcal{A}(J))$, which is the same as the $C^{*}$-subalgebra of $\mathcal{A}$ generated by $\mathcal{A}(I)$ and $\mathcal{A}(J)$, i.e. $\mathcal{A}(I \cup J)$, is isomorphic to a full matrix algebra of $d^{|I|} d^{|J|} \times d^{|I|} d^{|J|}$ matrices. This proves the first assertion.

Suppose $I$ is infinite. For an increasing sequence of finite subsets $L_{i}$ of $I$ tending to $I$, the union of $\mathcal{A}\left(L_{i}\right)$ generates $\mathcal{A}(I)$. Hence $\mathcal{A}(I)$ is the UHF algebra of type $d^{\infty}$.

Consequently, $\mathcal{A}(I)$ is simple for any $I$.

We need, in Section 4 and later, the following results. 
Theorem 2.2. For any countable family $\left\{I_{n}\right\}$ of subsets of $L$,

$$
\cap_{n=1}^{\infty} \mathcal{A}\left(I_{n}\right)=\mathcal{A}\left(\cap_{n=1}^{\infty} I_{n}\right)
$$

The proof is the same as that of Corollary 4.12 of [2], where we use Theorem 3.2 of Section 3.

Definition 2.1. For a subset $I$ of $L$,

$$
I_{-}=\left\{i \in I ;\left(\mathcal{A}_{i}\right)_{-} \neq 0\right\}
$$

namely $I_{-}$is the set of all $i \in I$ for which $\mathcal{A}_{i}$ has non-zero odd elements.

Note that if the lattice translation automorphisms $\tau_{k}$ exist, and $\Theta$ is non-trivial, then $\left(\mathcal{A}_{i}\right)_{-} \neq 0$ for all $i$ and $I_{-}=I$. The above notation is used in Sections 2 and 9 , where some results depend delicately on $I_{-}$and so they are stated in the situation with the translation uniformity assumption tentatively dropped in order to draw attention to the delicate situation.

Lemma 2.3. (1) For each $i \in L_{-}$, there exists a self-adjoint unitary $u_{i} \in\left(\mathcal{A}_{i}\right)_{+}$ implementing $\Theta$ on $\mathcal{A}_{i}$. It is unique up to \pm .

(2) If $I_{-}$is finite,

$$
u_{I}=\prod_{i \in I_{-}} u_{i}
$$

is a self-adjoint unitary in $\mathcal{A}(I)_{+}$implementing $\Theta$ on $\mathcal{A}(I)$, where the product is taken to be 1 if $I_{-}$is empty. Such $u_{I}$ is unique up to \pm .

(3) For each $i \in I_{-}$, there exists an even state $\omega_{i}$ of $\mathcal{A}_{i}$ satisfying $\omega_{i}\left(u_{i}\right)=0$

Proof. (1) This follows from the beginning part of the proof of Lemma 2.1.

(2) The first part follows from (1). The second part is due to the triviality of the center of $\mathcal{A}(I)$ given in Lemma 2.1.

(3) Since $u_{i}$ is a non-trivial self-adjoint unitary, $u_{i}=E_{i}^{+}-E_{i}^{-}$for mutually orthogonal non-trivial projections $E_{i}^{ \pm}$with sum 1. Set

$$
\begin{gathered}
\rho_{i}=\frac{1}{2}\left(\tau\left(E_{i}^{+}\right)^{-1} E_{i}^{+}+\tau\left(E_{i}^{-}\right)^{-1} E_{i}^{-}\right), \\
\omega_{i}(A)=\tau\left(\rho_{i} A\right), \quad\left(A \in \mathcal{A}_{i}\right) .
\end{gathered}
$$

Since $u_{i}$ is even, $E_{i}^{ \pm}$are even. Hence $\omega_{i}$ is even and satisfies $\omega_{i}\left(u_{i}\right)=0$.

Theorem 2.4. (1) If $I_{-}$is finite,

$$
\mathcal{A}(I)^{\prime} \cap \mathcal{A}=\mathcal{A}\left(I^{c}\right)_{+}+u_{I} \mathcal{A}\left(I^{c}\right)_{-}
$$

where $u_{I}$ is a self-adjoint unitary in $\mathcal{A}(I)$ implementing $\Theta$ on $\mathcal{A}(I)$, which exists.

(2) If $I_{-}$is infinite,

$$
\mathcal{A}(I)^{\prime} \cap \mathcal{A}=\mathcal{A}\left(I^{c}\right)_{+}
$$

The proof is the same as that of Theorem 4.17 of [2], except for two modifications. First we use a self-adjoint unitary $u_{I}$ given in Lemma 2.3. Second we apply the proof of Lemma 4.16 of [2] to the case of an infinite $I_{-}$, by using, instead of $E_{I}$ in [2], the conditional expectations $E_{I}^{\omega}$ in Section 3 for an even product state $\omega$ of the tracial state of $\mathcal{A}_{i}$ for $i \notin L_{-}$and the state $\omega_{i}$ given by Lemma 2.3 (3) for $i \in L_{-}$. 
Theorem 2.5. (1) If $I_{-}$is finite,

$$
\left(\mathcal{A}(I)_{+}\right)^{\prime} \cap \mathcal{A}=\mathcal{A}\left(I^{c}\right)+u_{I} \mathcal{A}\left(I^{c}\right) .
$$

(2) If $I_{-}$is infinite,

$$
\left(\mathcal{A}(I)_{+}\right)^{\prime} \cap \mathcal{A}=\mathcal{A}\left(I^{c}\right) .
$$

The proof is the same as that of Theorem 4.19 of [2] with the same modification as the proof of the preceding Theorem.

Lemma 2.6. Assume that $I_{-}$is infinite. Then any $u \in \mathcal{A}$ satisfying $u A=\Theta(A) u$ for all $A \in \mathcal{A}(I)$ is 0 . In particular, $\Theta$ on $\mathcal{A}(I)$ is outer.

Proof is the same as that of Lemma 4.20 of [2].

\section{Conditional Expectations}

Let $\omega$ be a state of $\mathcal{A}$ possessing the following property.

Product Property: For any disjoint subsets $I_{1}, \ldots, I_{k}$ of $L$ and for any $A_{i} \in$ $\mathcal{A}\left(I_{i}\right) \quad(i=1, \ldots, k)$,

$$
\omega\left(A_{1}, \ldots, A_{k}\right)=\omega\left(A_{1}\right) \ldots \omega\left(A_{k}\right) .
$$

This property for an arbitrary pair of two disjoint one-point subsets $(k=2$, $\left|I_{1}\right|=\left|I_{2}\right|=1$ ) implies (3.1) for the general case because each $\mathcal{A}\left(I_{i}\right)$ is generated by $\mathcal{A}_{l}, l \in I_{i}$. Such a state is called a product state and is denoted as

$$
\omega=\prod_{i \in L} \omega_{i}
$$

where $\omega_{i}$ is the restriction of $\omega$ to $\mathcal{A}_{i}$. It is uniquely determined by $\omega_{i}$. It is known ([1],Theorem 1) that such a product state for given $\omega_{i}, i \in L$, exists if and only if all $\omega_{i}$ with at most one exception are even, i.e.

$$
\omega_{i}\left(\Theta\left(A_{i}\right)\right)=\omega\left(A_{i}\right) \quad \text { for } A_{i} \in \mathcal{A}_{i}
$$

or equivalently

$$
\omega_{i}\left(A_{i}\right)=0 \quad \text { for } A_{i} \in\left(\mathcal{A}_{i}\right)_{-}
$$

for all but one $i \in L$.

The product state (3.2) is even if and only if all $\omega_{i}$ are even ([1],Theorem 1).

Throughout this paper, except in Section $9, \omega$ is assumed to be an even product state.

A typical even product state is the tracial state $\tau$ which can be characterized by the following tracial property (see Proposition 8.1):

$$
\tau(A B)=\tau(B A) \quad \text { for all } A, B \in \mathcal{A} .
$$

Another example is the Fock vacuum in the case of Fermion lattice systems (see Proposition 8.2).

Theorem 3.1. Let $\omega$ be an even product state.

(1) For any subset $I$ of $L$ and any $A \in \mathcal{A}$, there exists a unique $E_{I}^{\omega}(A) \in \mathcal{A}(I)$ satisfying

$$
\omega\left(B_{1} A B_{2}\right)=\omega\left(B_{1} E_{I}^{\omega}(A) B_{2}\right)
$$

for all $B_{1}, B_{2} \in \mathcal{A}(I)$. 
(2) The map $E_{I}^{\omega}$ from $A$ to $E_{I}^{\omega}(A)$ is a conditional expectation from $\mathcal{A}$ to $\mathcal{A}(I)$, namely the following holds.

(2-1) It is linear, *-preserving, positive and unital.

$(2-2)$ For $B_{1}, B_{2} \in \mathcal{A}(I)$,

$$
E_{I}^{\omega}\left(B_{1} A B_{2}\right)=B_{1} E_{I}^{\omega}(A) B_{2} .
$$

(2-3) It is a projection of norm 1.

$(2-4) \Theta E_{I}^{\omega}=E_{I}^{\omega} \Theta$.

(2-5) If $\omega$ is translation invariant, then

$$
\tau_{k} E_{I}^{\omega}=E_{I+k}^{\omega} \tau_{k}, \quad(n \in L) .
$$

(3) The following relation holds:

$$
E_{I}^{\omega} E_{J}^{\omega}=E_{J}^{\omega} E_{I}^{\omega}=E_{I \cap J}^{\omega}
$$

Namely, the following diagram is a commuting square.

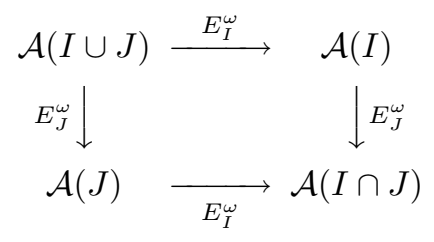

Before presenting the proof of this theorem, we give a result on continuity of $E_{I}^{\omega}$ on $I$. For any net $I_{\alpha}$ of subsets of $L, I_{\alpha} \rightarrow I$ means

$$
I=\cap_{\beta}\left(\cup_{\alpha \geq \beta} I_{\alpha}\right)=\cup_{\beta}\left(\cap_{\alpha \geq \beta} I_{\alpha}\right),
$$

the second equality being the condition for the convergence of the net $\left\{I_{\alpha}\right\}$. In particular, if $I_{\alpha}$ is monotone increasing, its limit is $I=\cup_{\alpha} I_{\alpha}$ and if $I_{\alpha}$ is monotone decreasing, its limit is $I=\cap_{\alpha} I_{\alpha}$, the convergence being automatic in both cases.

Theorem 3.2. If $I_{\alpha} \rightarrow I$, then

$$
\lim _{\alpha}\left\|E_{I_{\alpha}}^{\omega}(A)-E_{I}^{\omega}(A)\right\|=0
$$

for any $A \in \mathcal{A}$. In particular, if $I_{\alpha} \rightarrow L$, then

$$
\lim _{\alpha}\left\|E_{I_{\alpha}}^{\omega}(A)-A\right\|=0 \text {. }
$$

In other word,

$$
\lim _{I \rightarrow L} E_{I}^{\omega}=1
$$

The proof of this Theorem is exactly the same as that of Theorem 4.11 in [2]. The rest of this section is devoted to the proof of the first Theorem.

Lemma 3.3. If $E_{I}^{\omega}(A)$ satisfying (3.6) exists, then it is unique and

$$
\left\|E_{I}^{\omega}(A)\right\| \leq\|A\| \text {. }
$$

Proof. Consider the (GNS) triplet consisting of a Hilbert space $\mathcal{H}_{\omega}^{I}$, a representation $\pi_{\omega}^{I}$ of $\mathcal{A}(I)$ and a cyclic unit vector $\Omega_{\omega}^{I} \in \mathcal{H}_{\omega}^{I}$ giving rise to the restriction of the state $\omega$ to $\mathcal{A}(I)$. For $B_{1}, B, B_{2} \in \mathcal{A}(I)$,

$$
\begin{gathered}
\omega\left(B_{1} B B_{2}\right)=\left(\Psi_{1}, \pi_{\omega}^{I}(B) \Psi_{2}\right), \\
\Psi_{1}=\pi_{\omega}^{I}\left(B_{1}\right)^{*} \Omega_{\omega}^{I}, \quad \Psi_{2}=\pi_{\omega}^{I}\left(B_{2}\right) \Omega_{\omega}^{I},
\end{gathered}
$$

where $\left\{\Psi_{1} ; B_{1} \in \mathcal{A}(I)\right\}$ and $\left\{\Psi_{2} ; B_{2} \in \mathcal{A}(I)\right\}$ are dense in $\mathcal{H}_{\omega}^{I}$. 
If $B$ and $B^{\prime}$ in $\mathcal{A}(I)$ satisfy

$$
\omega\left(B_{1} B B_{2}\right)=\omega\left(B_{1} B^{\prime} B_{2}\right)
$$

for all $B_{1}$ and $B_{2}$ in $\mathcal{A}(I)$, then (3.15) implies

$$
\pi_{\omega}^{I}(B)=\pi_{\omega}^{I}\left(B^{\prime}\right) .
$$

Hence $B=B^{\prime}$ due to the simplicity of $\mathcal{A}(I)$. This proves the uniqueness.

Since $\left\|\Psi_{1}\right\|^{2}=\omega\left(B_{1} B_{1}^{*}\right)$ and $\left\|\Psi_{2}\right\|^{2}=\omega\left(B_{2}^{*} B_{2}\right)$, we obtain

$$
\left\|\pi_{\omega}^{I}(B)\right\|=\sup \left\{\left|\omega\left(B_{1} B B_{2}\right)\right| /\left(\omega\left(B_{1} B_{1}^{*}\right) \omega\left(B_{2}^{*} B_{2}\right)\right)^{1 / 2}\right\}
$$

where the sup is taken over all $B_{1}$ and $B_{2}$ in $\mathcal{A}(I)$ satisfying $\omega\left(B_{1} B_{1}^{*}\right) \neq 0$ and $\omega\left(B_{2}^{*} B_{2}\right) \neq 0$. The same formula as (3.15) and (3.16) for $I=L$ imply

$$
\left|\omega\left(B_{1} A B_{2}\right) /\left(\omega\left(B_{1} B_{1}^{*}\right) \omega\left(B_{2}^{*} B_{2}\right)\right)^{1 / 2}\right| \leq\left\|\pi_{\omega}^{L}(A)\right\| .
$$

Since $\mathcal{A}(I)$ and $\mathcal{A}$ are simple, we have

$$
\left\|\pi_{\omega}^{I}(B)\right\|=\|B\|, \quad\left\|\pi_{\omega}^{L}(A)\right\|=\|A\| .
$$

Hence the above two relations imply (for $B=E_{I}^{\omega}(A)$ )

$$
\left\|E_{I}^{\omega}(A)\right\| \leq\|A\| \text {. }
$$

The following Lemma obviously holds.

Lemma 3.4. If $E_{I}^{\omega}\left(A_{1}\right)$ and $E_{I}^{\omega}\left(A_{2}\right)$ satisfying (3.6) exist, then $E_{I}^{\omega}\left(c_{1} A_{1}+c_{2} A_{2}\right)$ satisfying (3.6) exists and is given by

$$
E_{I}^{\omega}\left(c_{1} A_{1}+c_{2} A_{2}\right)=c_{1} E_{I}^{\omega}\left(A_{1}\right)+c_{2} E_{I}^{\omega}\left(A_{2}\right) .
$$

Proof of Theorem 3.1

(1)

First we consider

$$
A=B C, \quad B \in \mathcal{A}(I), \quad C \in \mathcal{A}\left(I^{c}\right)
$$

where $I^{c}$ denotes the complement of $I$ in $L$. We claim that

$$
E_{I}^{\omega}(A)=\omega(C) B
$$

satisfies (3.6). It is enough to check (3.6) for $B_{1} \in \mathcal{A}(I)_{\sigma_{1}}$ and $B_{2} \in \mathcal{A}(I)_{\sigma_{2}}$ for all choices of $\sigma_{1}= \pm$ and $\sigma_{2}= \pm$. By the decompositon (2.4), we have $C=C_{+}+C_{-}$ with $C_{\sigma} \in \mathcal{A}\left(I^{c}\right)_{\sigma}(\sigma= \pm)$ and it is enough to check (3.6) for $C_{\sigma}, \sigma= \pm$ instead of C.

We have

$$
\begin{aligned}
\omega\left(B_{1} A B_{2}\right) & =\omega\left(B_{1} B C_{\sigma} B_{2}\right)=\epsilon\left(\sigma, \sigma_{2}\right) \omega\left(B_{1} B B_{2} C_{\sigma}\right) \\
& =\epsilon\left(\sigma, \sigma_{2}\right) \omega\left(B_{1} B B_{2}\right) \omega\left(C_{\sigma}\right) \\
& =\epsilon\left(\sigma, \sigma_{2}\right) \omega\left(B_{1} E_{I}^{\omega}(A) B_{2}\right) .
\end{aligned}
$$

If $\sigma=-$, then $\omega\left(C_{\sigma}\right)=0$ because $\omega$ is even. Hence, (3.6) holds. If $\sigma=+$, then $\epsilon\left(\sigma, \sigma_{2}\right)=1$ irrespective of $\sigma_{2}$ and (3.6) holds. So (3.24) satisfies (3.6).

By the graded commutation relations between elements of $\mathcal{A}(I)$ and $\mathcal{A}\left(I^{c}\right)$, any polynomial of a finite number of elements in $\mathcal{A}(I)$ and $\mathcal{A}\left(I^{c}\right)$ can be written as a linear combination of the product (3.23). By Lemma 3.4, $E_{\omega}^{I}(A)$ satisfying (3.6) exists for any element $A$ in the algebraic span of $\mathcal{A}(I)$ and $\mathcal{A}\left(I^{c}\right)$. 
If $A_{n}$ is a Cauchy sequence tending to $A$, and $E_{I}^{\omega}\left(A_{n}\right)$ exists for all $n$, then $E_{I}^{\omega}\left(A_{n}\right)$ is a Cauchy sequence by Lemma 3.4 and Lemma 3.3. Hence the limit

$$
E_{I}^{\omega}(A)=\lim _{n} E_{I}^{\omega}\left(A_{n}\right) \in \mathcal{A}(I)
$$

exists in $\mathcal{A}(I)$ and satisfies (3.6). This proves the existence of $E_{I}^{\omega}$ for all $A \in \mathcal{A}$.

The uniqueness of $E_{I}^{\omega}(A)$ is already given by Lemma 3.3 .

(2)

(2-1) The linearity is given by Lemma 3.4. By

$$
\begin{aligned}
\omega\left(B_{1} E_{I}^{\omega}(A)^{*} B_{2}\right) & =\overline{\omega\left(B_{2}^{*} E_{I}^{\omega}(A) B_{1}^{*}\right)}=\overline{\omega\left(B_{2}^{*} A B_{1}^{*}\right)}=\omega\left(B_{1} A^{*} B_{2}\right) \\
& =\omega\left(B_{1} E_{I}^{\omega}\left(A^{*}\right) B_{2}\right),
\end{aligned}
$$

we obtain $E_{I}^{\omega}(A)^{*}=E_{I}^{\omega}\left(A^{*}\right)$. By (3.15) and (3.16) with $B_{1}=B_{2}^{*}$, we have

$$
\begin{aligned}
\left(\Psi_{2}, \pi_{\omega}^{I}\left(E_{I}^{\omega}\left(A^{*} A\right)\right) \Psi_{2}\right) & =\omega\left(B_{2}^{*} E_{I}^{\omega}\left(A^{*} A\right) B_{2}\right)=\omega\left(B_{2}^{*} A^{*} A B_{2}\right) \\
& \geq 0 .
\end{aligned}
$$

This implies $\pi_{\omega}\left(E_{I}^{\omega}\left(A^{*} A\right)\right) \geq 0$ and hence $E_{I}^{\omega}\left(A^{*} A\right) \geq 0$ by the faithfulness of $\pi_{\omega}$ (due to the simplicity of $\mathcal{A}(I)$ ). Finally $E_{I}^{\omega}(1)=1 \in \mathcal{A}(I)$ satisfies (3.6) and hence $E_{I}^{\omega}$ is unital.

(2-2) If $B_{1}, B_{2}, B_{1}^{\prime}, B_{2}^{\prime \prime} \in \mathcal{A}(I)$, then

$$
\begin{aligned}
\omega\left(B_{1}^{\prime} B_{1} E_{I}^{\omega}(A) B_{2} B_{2}^{\prime}\right) & =\omega\left(B_{1}^{\prime} B_{1} A B_{2} B_{2}^{\prime}\right) \\
& =\omega\left(B_{1}^{\prime} E_{I}^{\omega}\left(B_{1} A B_{2}\right) B_{2}^{\prime}\right) .
\end{aligned}
$$

The uniqueness and $B_{1} E_{I}^{\omega}(A) B_{2} \in \mathcal{A}(I)$ implies (2-2).

(2-3) Since $E_{I}^{\omega}$ is unital, (2-2) implies $E_{I}^{\omega}(B)=B$ if $B \in \mathcal{A}(I)$. Hence

$$
E_{I}^{\omega}\left(E_{I}^{\omega}(A)\right)=E_{I}^{\omega}(A),
$$

namely $E_{I}^{\omega}$ is a projection. Lemma 3.3 and $E_{I}^{\omega}(1)=1$ imply $\left\|E_{I}^{\omega}\right\|=1$.

(2-4) Since $\omega$ is even, we have

$$
\begin{aligned}
\omega\left(B_{1} E_{I}^{\omega}(\Theta(A)) B_{2}\right) & =\omega\left(B_{1} \Theta(A) B_{2}\right) \\
& =\omega\left(\Theta\left(B_{1} \Theta(A) B_{2}\right)\right)=\omega\left(\Theta\left(B_{1}\right) A \Theta\left(B_{2}\right)\right) \\
& =\omega\left(\Theta\left(B_{1}\right) E_{I}^{\omega}(A) \Theta\left(B_{2}\right)\right)=\omega\left(\Theta\left(\Theta\left(B_{1}\right) E_{I}^{\omega}(A) \Theta\left(B_{2}\right)\right)\right) \\
& =\omega\left(B_{1} \Theta\left(E_{I}^{\omega}(A)\right) B_{2}\right) .
\end{aligned}
$$

By uniqueness, we have

$$
E_{I}^{\omega}(\Theta(A))=\Theta\left(E_{I}^{\omega}(A)\right) .
$$

(2-5) Due to $\tau_{k}(\mathcal{A}(I))=\mathcal{A}(I+k)$, we have for $B_{1}, B_{2} \in \mathcal{A}(I+k)$

$$
\begin{aligned}
\omega\left(B_{1} E_{I+k}^{\omega}\left(\tau_{k}(A)\right) B_{2}\right) & =\omega\left(B_{1} \tau_{k}(A) B_{2}\right)=\omega\left(\tau_{-k}\left(B_{1} \tau_{k}(A) B_{2}\right)\right) \\
& =\omega\left(\tau_{-k}\left(B_{1}\right) A \tau_{-k}\left(B_{2}\right)\right)=\omega\left(\tau_{-k}\left(B_{1}\right) E_{I}^{\omega}(A) \tau_{-k}\left(B_{2}\right)\right) \\
& =\omega\left(\tau_{k}\left(\tau_{-k}\left(B_{1}\right) E_{I}^{\omega}(A) \tau_{-k}\left(B_{2}\right)\right)\right) \\
& =\omega\left(B_{1} \tau_{k}\left(E_{I}^{\omega}(A)\right) B_{2}\right)
\end{aligned}
$$

where $\tau_{-k}\left(B_{1}\right), \tau_{-k}\left(B_{2}\right) \in \mathcal{A}(I)$. Hence $E_{I+k}^{\omega}\left(\tau_{k}(A)\right)=\tau_{k}\left(E_{I}^{\omega}(A)\right)$.

(3)

If $A \in \mathcal{A}(K)$ in the proof of (1), it is enough to take $B \in \mathcal{A}(I \cap K)$ and $C \in \mathcal{A}\left(I^{c} \cap K\right)$ due to $\mathcal{A}(K)=\mathcal{A}\left((I \cap K) \cup\left(I^{c} \cap K\right)\right)$. Hence we have

$$
E_{I}^{\omega}(A) \in \mathcal{A}(I \cap K)
$$


if $A \in \mathcal{A}(K)$. On the other hand, for $B_{1}, B_{2} \in \mathcal{A}(I \cap J)$,

$$
\begin{aligned}
\omega\left(B_{1} E_{I \cap J}^{\omega}(A) B_{2}\right) & =\omega\left(B_{1} A B_{2}\right)=\omega\left(B_{1} E_{J}^{\omega}(A) B_{2}\right) \\
& =\omega\left(B_{1} E_{I}^{\omega}\left(E_{J}^{\omega}(A)\right) B_{2}\right)
\end{aligned}
$$

where the second equality is due to $B_{1}, B_{2} \in \mathcal{A}(J)$ and the third due to $B_{1}, B_{2} \in$ $\mathcal{A}(I)$. Hence $E_{I \cap J}^{\omega}=E_{I}^{\omega} E_{J}^{\omega}$ by uniqueness. By interchanging the role of I and $\mathrm{J}$, we also obtain $E_{I \cap J}^{\omega}=E_{J}^{\omega} E_{I}^{\omega}$.

\section{4. $\omega$-STANDARD POTENTIAL}

We use notation in [2]. We start with the real vector space $\Delta\left(\mathcal{A}_{0}\right)$ of all *derivations $\delta$ with domein $\mathcal{A}_{0}$ and commuting with $\Theta$.

If a dynamics $\alpha_{t}$ of $\mathcal{A}$ (i.e. a continuous one-parameter group of automorphisms) satisfies

Assumption I : $\Theta \alpha_{t}=\alpha_{t} \Theta$,

Assumption II : The domain of the generator $\delta_{\alpha}$ of $\alpha_{t}$ contains $\mathcal{A}_{0}$, then the restriction of $\delta_{\alpha}$ to $\mathcal{A}_{0}$ is in $\Delta\left(\mathcal{A}_{0}\right)$.

We consider the real vector space $\mathcal{H}^{\omega}$ of functions $H^{\omega}$ of finite subsets $I$ of $L$ with values $H^{\omega}(I)$ in $\mathcal{A}$ satisfying the following properties. (The vector space structure is taken to be that of a function space with values in a vector space.)

$(\mathrm{H}-1)_{\omega} H^{\omega}(I)^{*}=H^{\omega}(I) \in \mathcal{A}$,

$(\mathrm{H}-2)_{\omega} \Theta\left(H^{\omega}(I)\right)=H^{\omega}(I)$ (i.e. $\left.H^{\omega}(I) \in \mathcal{A}_{+}\right)$,

$(\mathrm{H}-4)_{\omega} E_{I^{c}}^{\omega}\left(H^{\omega}(I)\right)=0$,

$(\mathrm{H}-5)_{\omega} H^{\omega}(I)=H^{\omega}(J)-E_{I^{c}}^{\omega}\left(H^{\omega}(J)\right)$ for $I \subset J \subset \subset L$.

Theorem 4.1. The following relation between $H^{\omega} \in \mathcal{H}^{\omega}$ and $\delta \in \Delta\left(\mathcal{A}_{0}\right)$ gives a bijective, real linear map from $\mathcal{H}^{\omega}$ to $\Delta\left(\mathcal{A}_{0}\right)$.

$(\mathrm{H}-3)_{\omega} \quad \delta A=i\left[H^{\omega}(I), A\right] \quad(A \in \mathcal{A}(I))$.

The proof is the same as that of Theorem 5.7 in [2]. The operator $H^{\omega}(I)$ will be called the $\omega$-standard local Hamiltonian for the region $I$ (for a given $\delta$ ).

The internal energy is defined by

$$
U^{\omega}(I)=E_{I}^{\omega}\left(H^{\omega}(I)\right) \quad(A \in \mathcal{A}(I)) .
$$

The local Hamiltonians $H^{\omega}(I)$ are recovered from the family $\left\{U^{\omega}(I)\right\}$ as follows.

$$
H^{\omega}(I)=\lim _{J \nearrow L}\left\{U^{\omega}(J)-E_{I^{c}}^{\omega}\left(U^{\omega}(J)\right)\right\} .
$$

Definition 4.1. A function $\Phi_{\omega}$ of a finite subset of $L$ with values in $\mathcal{A}$ is called an $\omega$-standard potential if it satisfies the following conditions.

$(\Phi-a) \quad \Phi^{\omega}(I) \in \mathcal{A}(I), \quad \Phi^{\omega}(\emptyset)=0$.

$(\Phi-b) \quad \Phi^{\omega}(I)^{*}=\Phi^{\omega}(I)$.

$(\Phi-c) \quad \Theta\left(\Phi^{\omega}(I)\right)=\Phi^{\omega}(I)$.

$(\Phi-d) \quad E_{J}^{\omega}\left(\Phi^{\omega}(I)\right)=0$ if $J \subset I$ and $J \neq I$.

$(\Phi-e) \quad$ For each $I \subset \subset L$. the net

$$
H_{J}^{\omega}(I)=\sum_{K}\left\{\Phi^{\omega}(K) ; K \cap I \neq \emptyset, K \subset J\right\}
$$

is a Cauchy net in the norm topology of $\mathcal{A}$ for $J \rightarrow L$. (The index set of the net is the family of all finite subsets $J$ of $L$ partially ordered by the set inclusion.) 
The vector space of all $\omega$-standard potentials is denoted by $\mathcal{P}^{\omega}$.

Remark 4.1. The condition $(\Phi-d)$ is equivalent to the following condition due to $(\Phi-a)$.

$$
(\Phi-d)^{\prime} \quad E_{J}^{\omega}\left(\Phi^{\omega}(I)\right)= \begin{cases}\Phi^{\omega}(I), & \text { if } I \subset J \\ 0, & \text { otherwise }\end{cases}
$$

(See Remark to Definition 5.10 in [2].)

Theorem 4.2. (1) The following equation gives a bijective, real linear map from $\Phi^{\omega} \in \mathcal{P}^{\omega}$ to $H^{\omega} \in \mathcal{H}^{\omega}$

$$
H^{\omega}(I)=\lim _{J \nearrow L} \sum_{K}\left\{\Phi^{\omega}(K) ; K \cap I \neq \emptyset, K \subset J\right\} .
$$

(2) The relations $(\mathrm{H}-3)_{\omega}$ and (4.4) give a bijective, real linear map from $\Phi^{\omega} \in \mathcal{P}^{\omega}$ to $\delta \in \Delta\left(\mathcal{A}_{0}\right)$.

Remark 4.2. The following relations hold.

$$
\begin{gathered}
U^{\omega}(I)=\sum_{K \subset I} \Phi^{\omega}(K), \\
\Phi^{\omega}(I)=\sum_{K \subset I}(-1)^{|I|-|K|} U^{\omega}(K) .
\end{gathered}
$$

The proof of these Theorem and Remark are the same as those of Theorems 5.12 and 5.13 in $[2]$.

\section{GibBS And LTS CONDITIONS}

We call a function $\Phi$ of a finite subset $I$ of $L$ with values $\Phi(I)$ in $\mathcal{A}$ a general potential if the conditions $(\Phi-a),(\Phi-b),(\Phi-c)$ and $(\Phi-e)$ of Definition 4.1 are satisfied (where $\Phi^{\omega}$ there is replaced by $\Phi$ ). Then the relations

$$
\begin{gathered}
H_{\Phi}(I)=\lim _{J \nearrow L} \sum\{\Phi(K) ; K \cap I \neq \emptyset, K \subset J\}, \\
\delta_{\Phi}(A)=i\left[H_{\Phi}(I), A\right], \quad(A \in \mathcal{A}(I))
\end{gathered}
$$

defines $\delta_{\Phi} \in \Delta\left(\mathcal{A}_{0}\right)$ consistently. Consistency means

$$
\left[H_{\Phi}(I), A\right]=\left[H_{\Phi}(J), A\right]
$$

if $A \in \mathcal{A}(I) \cap \mathcal{A}(J)(=\mathcal{A}(I \cap J))$.

If $\delta_{\Phi_{1}}=\delta_{\Phi_{2}}$, then $\Phi_{1}$ and $\Phi_{2}$ are said to be equivalent. For a given $\delta \in \Delta\left(\mathcal{A}_{0}\right)$, the corresponding $\omega$-standard potential $\Phi^{\omega}$ satisfies $\delta_{\Phi^{\omega}}=\delta$ and hence $\Phi^{\omega}$ for different $\omega$ 's and for a fixed $\delta$ are equivalent potentials.

We shall now check that each of Gibbs and LTS conditions, which are possible characterization of equiblibrium states, are mutually equivalent for equivalent potentials.

Definition 5.1. A state $\varphi$ of $\mathcal{A}$ satisfies $(\Phi, \beta)$-Gibbs condition for a general potential $\Phi$ and $\beta \in \mathbb{R}$ if the following two conditions hold.

(1) It is modular (i.e. its extention to the weak closure $\pi_{\varphi}(\mathcal{A})^{\prime \prime}$ of the (GNS) cyclic representation $\pi_{\varphi}$ of $\mathcal{A}$ is separating).

(2) The (GNS) representing operators $\pi_{\varphi}(\mathcal{A}(I))$ is in the centralizer of the perturbed functional $\varphi^{h}$ for the perturbation $h=\beta H_{\Phi}(I)$, i.e. they are elementwise invariant under the modular automorphism group of $\varphi^{h}$. Equivalently,

$$
\varphi^{h}(A B)=\varphi^{h}(B A)
$$

for any $A \in \mathcal{A}(I)$ and any $B \in \mathcal{A}$. 
(See Definition 7.1 with conditions (D-1) and (D-2)' in [2])

If $\Phi_{1}$ and $\Phi_{2}$ are equivalent general potentials, we have

$$
\left[H_{\Phi_{1}}(I), A\right]=\left[H_{\Phi_{2}}(I), A\right]
$$

for all $A \in \mathcal{A}(I)$ and hence

$$
\Delta H=\beta H_{\Phi_{1}}(I)-\beta H_{\Phi_{2}}(I) \in \mathcal{A}(I)^{\prime} .
$$

For $\varphi_{i}=\varphi^{H_{i}}$ where $H_{i}=\beta H_{\Phi_{i}}(I)$, we have

$$
\varphi_{2}=\left(\varphi_{1}\right)^{-\Delta H}, \quad \varphi_{1}=\left(\varphi_{2}\right)^{\Delta H} .
$$

Hence, for modular automorphisms $\sigma_{t}^{\varphi_{i}}$, we have

$$
\begin{aligned}
\frac{d}{d t} \sigma_{t}^{\varphi_{1}}\left(\pi_{\varphi}(A)\right) & =\frac{d}{d t} \sigma_{t}^{\varphi_{2}}\left(\pi_{\varphi}(A)\right)+i \pi_{\varphi}([\Delta H, A]) \\
& =\frac{d}{d t} \sigma_{t}^{\varphi_{2}}\left(\pi_{\varphi}(A)\right)
\end{aligned}
$$

for $A \in \mathcal{A}(I)$. Therefore the vanishing of $\frac{d}{d t} \sigma_{t}^{\varphi_{i}}\left(\pi_{\varphi}(A)\right)$, which is necesary and sufficient for the validity of the condition (2) of the Gibbs condition for $\varphi_{i}$, is equivalent for $i=1$ and $i=2$. This proves that a state satisfies the $\left(\Phi_{1}, \beta\right)$-Gibbs condition if and only if it satisfies the $\left(\Phi_{2}, \beta\right)$-Gibbs condition.

Definition 5.2. (1)A state $\varphi$ satisfies the $(\Phi, \beta)$-LTSM condition if

$$
\tilde{S}_{I}^{M}(\varphi)-\beta \varphi\left(H_{\Phi}(I)\right) \geq \tilde{S}_{I}^{M}(\psi)-\beta \psi\left(H_{\Phi}(I)\right)
$$

for each finite subset $I$ and for all states $\psi$ with the same restriction to $\mathcal{A}(I)^{\prime}$ as the state $\varphi$

(2) A state $\varphi$ satisfies the $(\Phi, \beta)-L T S P$ condition if the above condition (5.10) with $M$ replaced by $P$ holds for all states $\psi$ which have the same restriction to $\mathcal{A}\left(I^{c}\right)$ as $\varphi$.

Here $\tilde{S}_{I}^{M}$ and $\tilde{S}_{I}^{P}$ are conditional entropy, independent of the potential. (See [3]; $M$ and $P$ refer to mathematical and physical.)

The equivalence of LTS conditions for equivalent general potentials is already obtained in Corollary 5 of [3] with its proof in Section 4 of [3].

Thus we have the following.

Theorem 5.1. Let $\Phi_{1}$ and $\Phi_{2}$ be equivalent general potentials.

(1) The $\left(\Phi_{i}, \beta\right)$-Gibbs conditions for $i=1$ and $i=2$ are equivalent.

(2) Each of $\left(\Phi_{i}, \beta\right)-L T S M$ conditions and $\left(\Phi_{i}, \beta\right)$-LTSP conditions for $i=1$ and $i=2$ are equivalent.

\section{Translation InVARIANCE}

A dynamics $\alpha_{t}$ is said to be translation invariant if the following holds.

Asumption IV : $\tau_{k} \alpha_{t}=\alpha_{t} \tau_{k}$ for any $t \in \mathbb{R}$ and $k \in L$.

This Assumption implies Assumption I in Section 4. (See Proposition 8.1 of [2].) A $*$-derivation $\delta \in \Delta\left(\mathcal{A}_{0}\right)$ is said to be translation invariant if $\tau_{k} \delta=\delta \tau_{k}$ for all $k \in L$. The real vector subspace of $\Delta\left(\mathcal{A}_{0}\right)$ consisting of all translation invariant $\delta \in \Delta\left(\mathcal{A}_{0}\right)$ will be denoted by $\Delta_{\tau}\left(\mathcal{A}_{0}\right)$. 
A potential $\Phi \in \mathcal{P}^{\omega}$ is said to be translation covariant if

$(\Phi-f) \quad \tau_{k}(\Phi(I))=\Phi(I+k) \quad$ for all $I \subset \subset L$ and $k \in L$.

The real linear subspace of $\mathcal{P}^{\omega}$ consisting of all translation covariant $\Phi \in \mathcal{P}^{\omega}$ is denoted by $\mathcal{P}_{\tau}^{\omega}$.

Theorem 6.1. The bijection of Theorem 4.2(2) maps $\Delta_{\tau}\left(\mathcal{A}_{0}\right)$ onto $\mathcal{P}_{\tau}^{\omega}$.

The proof is by a straightforward computation. (See Corollary 8.5 of [2].)

A potential $\Phi$ is said to be of finite range if there is a positive $d \in \mathbb{R}$ such that $\Phi(I)=0$ whenever the maximum distance of two points in $I$ exceeds $d$.

Theorem 6.2. With respect to

$$
\|\Phi\|:=\|\Phi(\{n\})\|, \quad \Phi \in \mathcal{P}_{\tau}^{\omega},
$$

which is independent of a point $n \in L$ and is a norm, $\mathcal{P}_{\tau}^{\omega}$ is a separable Banach space, in which the subspace of all finite range potentials is dense.

The proof is the same as those of Proposition 8.8, Proposition 8.12 and Corollary 8.13 .

The following energy estimates can be shown by the same proof as those of Lemmas 8.6 and 9.1 of [2], where

$$
\begin{aligned}
W^{\omega}(I) & =H^{\omega}(I)-U^{\omega}(I) \\
& =\lim _{J \rightarrow \infty} \sum\left\{\Phi^{\omega}(K) ; K \cap I \neq \emptyset, K \cap I^{c} \neq \emptyset, K \subset J\right\} .
\end{aligned}
$$

\section{Theorem 6.3.}

$$
\begin{gathered}
\left\|U^{\omega}(I)\right\| \leq\left\|H^{\omega}(I)\right\| \leq|I|\left\|\Phi^{\omega}\right\|, \\
\text { v.H. } \lim _{I \rightarrow \infty} \frac{1}{|I|}\left\|W^{\omega}(I)\right\|=0 .
\end{gathered}
$$

Here, v.H. $\lim _{I \rightarrow \infty}$ denotes the van Hove limit. (See Appendix of [2].)

\section{Variational Principle}

Theorem 7.1. For a translation invariant state $\varphi$ of $\mathcal{A}$ and $\Phi^{\omega} \in \mathcal{P}_{\tau}^{\omega}$, the following limits exist.

$$
\begin{aligned}
& p\left(\Phi^{\omega}\right)=\text { v.H. } \lim _{I \rightarrow \infty}|I|^{-1} \log \tau\left(e^{H^{\omega}(I)}\right)=\text { v.H. } \lim _{I \rightarrow \infty}|I|^{-1} \log \tau\left(e^{U^{\omega}(I)}\right) \\
& e_{\Phi \omega}(\varphi)=\text { v.H. } \lim _{I \rightarrow \infty} \varphi\left(H^{\omega}(I)\right) /|I|=\text { v.H. } \lim _{I \rightarrow \infty} \varphi\left(U^{\omega}(I)\right) /|I| \\
& \hat{s}(\varphi)=\text { v.H. } \lim _{I \rightarrow \infty} \hat{S}_{I}(\varphi) /|I|
\end{aligned}
$$

where $\hat{S}_{I}(\varphi)=-\tau\left(\hat{\rho}_{\varphi I} \log \hat{\rho}_{\varphi I}\right)$ for the adjusted density matrix $\hat{\rho}_{\varphi I}$ of the restriction of $\varphi$ to $\mathcal{A}(I)$, characterized by $\hat{\rho}_{\varphi I} \in \mathcal{A}(I)$ and $\varphi(A)=\tau\left(\hat{\rho}_{\varphi I} A\right)$ for all $A \in \mathcal{A}(I)$.

The proof is the same as those of Theorems 9.3, 9.5 and 10.3 in [2].

A translation invariant state $\varphi$ satisfies the $(\Phi, \beta)$ - variational principle if

$$
p(\beta \Phi)=\tilde{s}(\varphi)-\beta e_{\Phi}(\varphi) .
$$

By Proposition 14.1 of [2], $\varphi$ is a solution of (7.4) for a general potential $\Phi$ if and only if it is a solution of (7.4) for the $\tau$-standard potential $\Phi^{\tau}$ equivalent to $\Phi$, under the condition (14.2) and (14.3) of [2] for $\Phi$. For $\omega$-standard potential $\Phi^{\omega}$, 
the condition (14.2) of [2] is fullfiled due to (6.5) and the limit (14.3) of [2], being $e_{\Phi^{\omega}}(\tau)$, converges. Therefore the solutions of $\left(\Phi^{\omega}, \beta\right)$-variational principle coincide with those of $\left(\Phi^{\tau}, \beta\right)$-variational principle and hence the solution set is independent of $\omega$. Thus we have established the following result.

Theorem 7.2. For any pair of even product states $\omega$ and $\omega$ ', and for the $\omega$ - and $\omega$ 'standard potentials $\Phi^{\omega}$ and $\Phi^{\omega^{\prime}}$ corresponding to the same $\delta \in \Delta\left(\mathcal{A}_{0}\right)$, a translation invariant state $\varphi$ is a solution of the $\left(\Phi^{\omega}, \beta\right)$-variational principle if and only if it is a solution of the $\left(\Phi^{\omega^{\prime}}, \beta\right)$-variational principle.

\section{EXAMPLES OF EQUIVALENT $\omega$-STANDARD POTENTIALS FOR DIFFERENT $\omega$}

A potential $\Phi(I)$ belongs to $\mathcal{A}(J)$ if $J \supset I$. Hence a part of $\Phi(I)$ may be taken out and included in the potential $\Phi(J)$ without changing the dynamics (more specifically, without changing the corresponding derivation). This is the origin of the existence of equivalent potentials. In this section, we illustrate this by taking two different even product states $\omega$ and comparing the potentials $\Phi^{\omega}$ for the same derivation $\delta$.

The following two Propositions provide examples of even product states.

Proposition 8.1. The tracial state $\tau$ of $\mathcal{A}$ is an even product state.

Proof. The tracial state of $\mathcal{A}$ is unique and hence invariant under any automorphism. In particular it is even and $\tau(A)=0$ for any odd $A$.

Let $i \neq j$ and $A_{\sigma} \in\left(\mathcal{A}_{i}\right)_{\sigma}, B_{\sigma^{\prime}} \in\left(\mathcal{A}_{j}\right)_{\sigma^{\prime}} \quad\left(\sigma, \sigma^{\prime}= \pm\right)$. It is enough to show

$$
\tau\left(A_{\sigma} B_{\sigma^{\prime}}\right)=\tau\left(A_{\sigma}\right) \tau\left(B_{\sigma^{\prime}}\right)
$$

for all pair $i, j$ and all combinations of $\sigma= \pm$ and $\sigma^{\prime}= \pm$.

Consider the case $\sigma^{\prime}=-$ first. Then the right hand side of (8.1) vanishes. If $\sigma=+$, then the left hand side also vanishes because $A_{+} B_{-}$is odd. If $\sigma=-$, then

$$
\tau\left(A_{-} B_{-}\right)=\tau\left(B_{-} A_{-}\right)=-\tau\left(A_{-} B_{-}\right)=0
$$

due to the tracial property of $\tau$ and the anti-commutativity of $A_{-}$and $B_{-}$. Therefore, (8.1) holds when $\sigma^{\prime}=-$.

Consider the case $\sigma^{\prime}=+$. For any $A_{1}, A_{2} \in \mathcal{A}_{i}$,

$$
\tau\left(A_{1} A_{2} B_{+}\right)=\tau\left(A_{2} B_{+} A_{1}\right)=\tau\left(A_{2} A_{1} B_{+}\right)
$$

due to the tracial property of $\tau$ and the commutativity of $A_{1}$ and $B_{+}$. Hence

$$
\tau\left(\left[A_{1}, A_{2}\right] B_{+}\right)=0 .
$$

Since $\mathcal{A}_{i}$ is isomorphic to a full matrix algebra, its element $A$ is a sum of $\tau(A) \mathbf{1}$ and commutators of elements of $\mathcal{A}_{i}$. Hence (8.1) holds for the present case too.

Proposition 8.2. For Fermion algebras (the case where each $\mathcal{A}_{i}$ is generated by a finite number of Fermion creation and annihilation operators $a_{i \alpha}^{*}$ and $a_{i \alpha}$ ), the vacuum state $\omega_{0}$ (uniquely characterized by

$$
\omega_{0}\left(a_{i \alpha}^{*} a_{i \alpha}\right)=0
$$

for all $i$ and $\alpha)$ is an even product state. 
Proof. Let the restriction of $\omega_{0}$ to $\mathcal{A}_{i}$ be $\omega_{0 i}$. Since (8.5) is invariant under the transposed action $\omega \rightarrow \omega \Theta$ of $\Theta$ on states $\omega, \omega_{0}$ as well as all $\omega_{0 i}$ are even. Then $\prod_{i} \omega_{0 i}$ is a state of $\mathcal{A}$ satisfying (8.5) and hence coincides with $\omega_{0}$. Therefore, $\omega_{0}$ is a product state.

Example of Equivalent Potentials: Consider the algebra $\mathcal{A}$ generated by Fermion creation and annihilation operators $a_{i}^{*}$ and $a_{i}, i \in L$, which is studied in [2]. We give below the $\omega$-standard (one-body and two body) potentials for the same dynamics and two different even product states: $\omega=\tau$ (the tracial state) and $\omega=\omega_{0}$ (the Fermion vacuum state). They give examples of equivalent potentials caused by different choices of $\omega$.

(1) One-body $\omega$-standard potentials.

For $\omega=\tau$ :

$$
\Phi^{\tau}(\{i\})=c\left(a_{i}^{*} a_{i}-a_{i} a_{i}^{*}\right), \quad(c \neq 0) .
$$

For $\omega=\omega_{0}$ :

$$
\Phi^{\omega_{0}}(\{i\})=2 c a_{i}^{*} a_{i}, \quad(c \neq 0) .
$$

They are related by

$$
\Phi^{\tau}(\{i\})-\Phi^{\omega_{0}}(\{i\})=-2 c \mathbf{1} \in \mathcal{A}(\emptyset)=\mathbb{C} \mathbf{1} .
$$

Since a multiple of the identity operator does not give any contribution to its commutator and hence to the corresponding derivation, the above $\tau$-standard and $\omega_{0}$-standard one-body potentials are equivalent.

(2) Two-body $\omega$-standard potentials.

For $\omega=\tau$ : Let $i \neq j$.

$$
\begin{aligned}
\Phi^{\tau}(\{i, j\})= & c_{1}\left(a_{i} a_{j}-a_{i}^{*} a_{j}^{*}\right)+c_{2}\left(a_{i} a_{j}^{*}-a_{i}^{*} a_{j}\right) \\
& +c_{3}\left(a_{i} a_{i}^{*}-a_{i}^{*} a_{i}\right)\left(a_{j} a_{j}^{*}-a_{j}^{*} a_{j}\right) .
\end{aligned}
$$

For $\omega=\omega_{0}$ : Let $i \neq j$.

$$
\Phi^{\omega_{0}}(\{i, j\})=c_{1}\left(a_{i} a_{j}-a_{i}^{*} a_{j}^{*}\right)+c_{2}\left(a_{i} a_{j}^{*}-a_{i}^{*} a_{j}\right)+4 c_{3} a_{i}^{*} a_{i} a_{j}^{*} a_{j} .
$$

They are related by

$$
\begin{array}{r}
\Phi^{\tau}(\{i, j\})-\Phi^{\omega_{0}}(\{i, j\})=-2 c_{3} a_{j}^{*} a_{j}-2 c_{3} a_{i}^{*} a_{i}+c_{3} \mathbf{1} \\
=-c_{3}\left(a_{j}^{*} a_{j}-a_{j} a_{j}^{*}\right)-c_{3}\left(a_{i}^{*} a_{i}-a_{i} a_{i}^{*}\right)-c_{3} \mathbf{1} .
\end{array}
$$

Namely the difference is expressed as a sum of $\omega_{0}$-standard one-body potentials at lattice sites $i$ and $j$, and also as a sum of $\tau$-standard one-body potentials at lattice sites $i$ and $j$, both modulo multiples of the identity operator. Therefore, the above $\tau$-standard two-body potential is equivalent to the above $\omega_{0}$-standard two-body potential combined with $\omega_{0}$-standard one-body potentials at lattice sites $i$ and $j$, and conversely the $\omega_{0}$-standard two-body potential is equivalent to the $\tau$-standard two-body potential combined with $(-1)$ times $\tau$-standard one-body potentials at lattice sites $i$ and $j$. In the case of translation covariant potentials, we will have (covariantly related) $\tau$-standard two-body potentials at all shifted pairs $\{i+n, j+n\}$ of lattice sites, $n \in L$. They are then equivalent to (covariantly related) $\omega_{0}$-standard two-body potentials at all shifted pairs $\{i+n, j+n\}$ of lattice sites, $n \in L$ combined with twice $\omega_{0}$-standard one-body potentials at all lattice sites (twice because any site will appear as $i+n$ once and as $j+n$ another time). Similarly, (covariantly 
related) $\omega_{0}$-standard two-body potentials at all shifted pairs $\{i+n, j+n\}$ of lattice sites are equivalent to (covariantly related) $\tau$-standard two-body potentials at all shifted pairs $\{i+n, j+n\}$ of lattice sites combined with $(-2)$ times $\tau$-standard one-body potentials at all lattice sites.

\section{Non-even Product State and Conditional Expectations}

We give a necessary and sufficient condition for the extistence of $\omega$-conditional expectation when one of the factor state of $\omega$ is not even.

A state $\omega_{I}$ of $\mathcal{A}(I)$ will be called an eigenstate of $u \in \mathcal{A}(I)$ belonging to an eigenvalue $\lambda$ if

$$
\omega_{I}(A u)=\lambda \omega_{I}(A)
$$

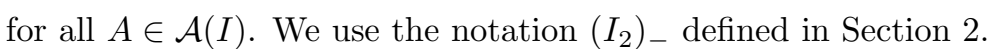

Theorem 9.1. Let $I_{1}$ and $I_{2}$ be mutually disjoint non-empty subset of $L$ and $I=$ $I_{1} \cup I_{2}$. Let $\omega_{i}$ be a state of $\mathcal{A}\left(I_{i}\right)(i=1,2)$ and a state $\omega$ of $\mathcal{A}(I)$ be a product state of $\omega_{1}$ and $\omega_{2}$. Assume that $\omega_{1}$ is not even.

(1) There exists the unique $\omega$-conditional expectation $E_{I_{1}}^{\omega}$ from $\mathcal{A}(I)$ onto $\mathcal{A}\left(I_{1}\right)$ in the sense of Theorem 3.1.

(2) No $\omega$-conditional expectation $E_{I_{2}}^{\omega}$ from $\mathcal{A}(I)$ onto $\mathcal{A}\left(I_{2}\right)$ in the sense of Theorem 3.1 exists if $\left(I_{2}\right)_{-}$is infinite.

(3) Assume that $\left(I_{2}\right)_{-}$is finite. Let $u_{2} \in \mathcal{A}\left(I_{2}\right)$ be a selfadjoint unitary implementing $\Theta$ restricted to $\mathcal{A}\left(I_{2}\right)$ (which exists). An $\omega$-conditional expectation $E_{I_{2}}^{\omega}$ from $\mathcal{A}(I)$ onto $\mathcal{A}\left(I_{2}\right)$ in the sense of Theorem 3.1 exists if and only if $\omega_{2}$ is an eigenstate of $u_{2}$. It is unique if it exists.

Proof. By theorem 1 of [1], $\omega_{2}$ must be even (in order that a product state of a non-even $\omega_{1}$ and $\omega_{2}$ exists).

(1) The proof of Theorem 3.1 goes through without any change.

(2) Let $A$ be an odd element of $\mathcal{A}\left(I_{1}\right)$ such that $\omega_{1}(A) \neq 0$. (Such an $A$ exists because $\omega_{1}$ is assumed to be not even.) Assuming that the $\omega$-conditional expectation $E_{I_{2}}^{\omega}$ from $\mathcal{A}(I)$ onto $\mathcal{A}\left(I_{2}\right)$ exists, we show a contradiction. Set $x=E_{I_{2}}^{\omega}(A)$. It has the following properties.

$(\alpha) x \neq 0$ because

$$
\omega(x)=\omega(A)=\omega_{1}(A) \neq 0 .
$$

(The first equality is due to (3.6) with $B_{1}=B_{2}=1$.)

( $\beta) x \in \mathcal{A}\left(I_{2}\right)$ by the defining property of $E_{I_{2}}^{\omega}$.

$(\gamma)$ By the property (2-2) of $E_{I_{2}}^{\omega}$ in Theorem 3.1, we have the following relation for any $B \in \mathcal{A}\left(I_{2}\right)$.

$$
\begin{aligned}
x B & =E_{I_{2}}^{\omega}(A) B=E_{I_{2}}^{\omega}(A B)=E_{I_{2}}^{\omega}(\Theta(B) A) \\
& =\Theta(B) E_{I_{2}}^{\omega}(A)=\Theta(B) x,
\end{aligned}
$$

where the third equality is by the graded commutation relations. By Lemma 2.6, $x=0$. This contradicts with $(\alpha)$.

(3) For sufficiency proof, assume that $\omega$ is an eigenstate of a selfadjoint unitary $u_{2} \in \mathcal{A}\left(I_{2}\right)$ which implements $\Theta$ on $\mathcal{A}\left(I_{2}\right)$. (Due to Lemma 2.3, the existence of such a $u_{2}$ follows from the assumption that $\left(I_{2}\right)_{-}$is finite.) Then the eigenvalue is either 1 or -1 due to $\left(u_{2}\right)^{2}=1$. By choosing $u_{2}$ from $\pm u_{2}$, we may assume that the eigenvalue is 1 . 
For $A_{\sigma}=B C_{\sigma}$ with $B \in \mathcal{A}\left(I_{2}\right), C_{\sigma} \in \mathcal{A}\left(I_{1}\right)_{\sigma}$, we set

$$
E_{I_{2}}^{\omega}\left(A_{+}\right)=\omega_{1}\left(C_{+}\right) B, \quad E_{I_{2}}^{\omega}\left(A_{-}\right)=\omega_{1}\left(C_{-}\right) B u_{2},
$$

and show that they satisfy (3.6) by the following computations, thereby showing that their linear combination gives an $\omega$-conditional expectation from $\mathcal{A}(I)$ onto $\mathcal{A}\left(I_{2}\right)$ due to Theorem 3.1.

$$
\begin{aligned}
\omega\left(B_{1} A_{+} B_{2}\right) & =\omega\left(B_{1} B B_{2} C_{+}\right)=\omega_{2}\left(B_{1} B B_{2}\right) \omega_{1}(C+) \\
& =\omega_{2}\left(B_{1}\left(\omega_{1}\left(C_{+}\right) B\right) B_{2}\right) \\
\omega\left(B_{1} A_{-} B_{2}\right) & =\omega\left(B_{1} B C_{-} B_{2}\right)=\omega\left(B_{1} B \Theta\left(B_{2}\right) C_{-}\right) \\
& =\omega_{2}\left(B_{1} B u_{2} B_{2} u_{2}\right) \omega_{1}\left(C_{-}\right)=\omega_{2}\left(B_{1}\left(\omega_{1}\left(C_{-}\right) B u_{2}\right) B_{2}\right)
\end{aligned}
$$

where the last equality is due to the assumption that $\omega_{2}$ is an eigenstate of $u_{2}$ belonging to an eigenvalue 1 .

For necessity proof, assume that $E_{I_{2}}^{\omega}$ exists. Let $C_{-} \in \mathcal{A}\left(I_{1}\right)_{-}$be such that $\omega_{1}\left(C_{-}\right) \neq 0$ and set $x=E_{I_{2}}^{\omega}\left(C_{-}\right)$. It satisfies the properties $(\alpha),(\beta)$, and $(\gamma)$ (except for the conclusion $x=0$ of $(\gamma)$ ) in the proof of $(2)$. In particular, $(\gamma)$ implies that $u_{2} x \in \mathcal{A}\left(I_{2}\right)$ commutes with all $B \in \mathcal{A}\left(I_{2}\right)$ and hence belongs to the center of $\mathcal{A}\left(I_{2}\right)$, which is trivial by Lemma 2.1. Hence $u_{2} x=c \mathbf{1}$ and $x=c u_{2}$ for some scalar $c$. By the same computation as in the sufficiency proof, we obtain the following relation for any $B_{1}, B_{2} \in \mathcal{A}\left(I_{2}\right)$.

$$
\begin{aligned}
\omega_{2}\left(B_{1} x B_{2}\right) & =\omega\left(B_{1} C_{-} B_{2}\right)=\omega\left(B_{1} \Theta\left(B_{2}\right) C_{-}\right) \\
& =\omega_{2}\left(B_{1} u_{2} B_{2} u_{2}\right) \omega_{1}\left(C_{-}\right) \\
& =\omega_{2}\left(B_{1} \omega_{1}\left(C_{-}\right) u_{2} B_{2} u_{2}\right) .
\end{aligned}
$$

Since $x=c u_{2}$, we have

$$
\omega_{1}\left(C_{-}\right)=\omega\left(C_{-}\right)=\omega_{2}(x)=c \omega_{2}\left(u_{2}\right) .
$$

By $\omega_{1}\left(C_{-}\right) \neq 0, c \neq 0$. Hence the equation $(9.4)=(9.5)$ with $B_{2}=1$ and the equation (9.7) give

$$
\omega_{2}\left(B_{1} u_{2}\right)=\omega_{2}\left(B_{1}\right) \omega_{2}\left(u_{2}\right)
$$

for all $B_{1} \in \mathcal{A}\left(I_{2}\right)$. Hence $\omega_{2}$ is an eigenstate of $u_{2}$.

Acknowledgement. This work has been completed during a visit to the University of Florida, made possible through the financial support of the Institute of Fundamental Theory. The author gratefully acknowledge hospitality by members of Department of Physics and Department of Mathematics, in particular by Professor John Klauder and by Professor Gerald Emch.

\section{REFERENCES}

[1] H.Araki and H.Moriya, Joint extension of states of subsystems for a CAR system, Commun.Math.Phys. 237(2003), 105-122.

[2] H.Araki and H.Moriya, Equilibrium statistical mechanics of Fermion lattice systems, Rev.Math.Phys. 15 (2003), 93-198.

[3] H.Araki and H.Moriya, Local thermodynamical stability of Fermion lattice systems, Lett.Math.Phys.62(2002), 33-45. 